\title{
Examining the identity of Yukawa with gauge couplings in supersymmetric QCD at LHC
}

\author{
Ayres Freitas, ${ }^{a}$ Peter Skands, ${ }^{b}$ Michael Spira ${ }^{c}$ and Peter M. Zerwas ${ }^{d}$ \\ ${ }^{a}$ Institut für Theoretische Physik, Universität Zürich, \\ Winterthurerstrasse 190, CH-805\%, Zürich, Switzerland \\ ${ }^{b}$ Theoretical Physics, Fermi National Accelerator Laboratory, \\ P.O. Box 500, Batavia, IL-60510, U.S.A. \\ ${ }^{c}$ Paul Scherrer Institut, \\ CH-5232 Villigen PSI, Switzerland \\ ${ }^{d}$ Deutsches Elektronensynchrotron DESY, \\ D-22603 Hamburg, Germany \\ E-mail: afreitas@physik.unizh.ch, skands@fnal.gov, michael.spira@psi.ch, \\ zerwas@desy.de
}

ABSTRACT: The identity of the quark-squark-gluino Yukawa coupling with the corresponding quark-quark-gluon QCD coupling in supersymmetric theories can be examined experimentally at the Large Hadron Collider (LHC). Extending earlier investigations of like-sign di-lepton final states, we include jets in the analysis of the minimal supersymmetric standard model, adding squark-gluino and gluino-pair production to squark-pair production. Moreover we expand the method towards model-independent analyses which cover more general scenarios. In all cases, squark decays to light charginos and neutralinos persist to play a dominant role.

KeYWords: Supersymmetry Phenomenology, Supersymmetric Standard Model, Beyond Standard Model. 


\section{Contents}

1. Introduction 1

2. Squark and gluino production at the LHC 2

3. Di-lepton plus jet analyses in the MSSM 3

4. Towards model-independent analyses 9

4.1 MSSM plus heavy iso-singlets 9

4.2 Generalised picture 12

5. Conclusions 14

\section{Introduction}

A characteristic consequence of supersymmetry is the identity of the quark-squark-gluino Yukawa coupling with the quark-quark-gluon coupling in SUSY-QCD [1]:

$$
\hat{g}_{\mathrm{s}}(q \tilde{q} \tilde{g})=g_{\mathrm{s}}(q q g)
$$

which persists to hold after the supersymmetry is broken softly. Similar identities are predicted in other sectors of the supersymmetric extension of the Standard Model.

Pair production of sleptons and charginos/neutralinos in $e^{+} e^{-}$collisions has been investigated to test the identity of Yukawa with gauge couplings in the non-coloured electroweak sector, $c f$. refs. 造- The equality of electroweak Yukawa and gauge couplings is expected to be studied at the per-cent to per-mil level by these experiments. In a recent report, ref. [5], the measurement of like-sign di-leptons has been examined to study the squark-pair production processes $q q \rightarrow \tilde{q} \tilde{q}$. Since these processes are mediated solely by the exchange of gluinos, they provide, in principle, the most appealing measurement of the Yukawa coupling in supersymmetric QCD. All branching ratios in the decay cascade $\tilde{q} \rightarrow \tilde{\chi}_{1}^{ \pm} \rightarrow \tilde{\ell}$ have been assumed to be known in evaluating the $\tilde{q} \tilde{q}$ pair cross section which involves the Yukawa coupling.

In this note we extend the analysis in two directions. First we will include the measurement of jets in like-sign di-lepton events, motivated by the large production cross sections for gluinos [6] which significantly enlarge the like-sign di-lepton ensemble through instantaneous decays to real or virtual squarks [see also ref. [7]]. In a second step we will investigate experimental scenarios in which only the properties of light gaugino-type charginos and neutralinos have been pre-determined either at LHC or at an $e^{+} e^{-}$linear collider [8]. This method, within a wide variety of theories, does not rely, in particular, on 
the measurement of the decay branching ratios of heavy squarks. Instead of studying the absolute size of the cross sections, we will analyse ratios of like-sign di-lepton cross sections and the evolution of these cross sections with the transverse momenta of additional jets. Including jets with varying transverse momenta in the analysis changes the relative weight of the subprocesses with different dependence on the Yukawa coupling. Therefore, relating the cross sections for different final state configurations, the overall normalisation can be eliminated from the analysis and the Yukawa coupling can be determined without a priori knowledge of the branching ratios for $\tilde{q}$ decays.

\section{Squark and gluino production at the LHC}

Most suited for this purpose, in the area of parameter space in which gluinos are heavier than squarks, are the basic subprocesses

$$
\begin{aligned}
\text { squark-pair production }: q q & \rightarrow \tilde{q} \tilde{q}(g) \\
\text { Compton process }: g q & \rightarrow \tilde{q} \tilde{g}(g) \text { with } \tilde{g} \rightarrow \tilde{q} q
\end{aligned}
$$

cf. figure 1. Gluinos decay instantly to squarks under this kinematical condition. $(g)$ denotes additional gluon jets emitted in the production process. The analysis of this kinematical area, admittedly, is easier than the reverse mass configuration requiring control of more extended cascade decays, but it is sufficient to illustrate the basic points. Other processes of secondary importance, like gluino-gluino pair production etc., have been included in the final analysis properly. As evident from figure 1, the size of the cross section for squark-pair production is set by the fourth power of the strong Yukawa coupling, $\hat{g}_{\mathrm{s}}^{4}$, while the cross section of the Compton process is determined by the product of the second power of the gauge and Yukawa couplings, $g_{\mathrm{s}}^{2} \hat{g}_{\mathrm{s}}^{2}$. Since the relative weight of the subprocesses changes for different jet final-state configurations, the Yukawa coupling can be measured by comparing the cross sections in different parts of the phase space.

To focus on the basic processes eqs. (2.1), 2.2) and to reduce the backgrounds from other SUSY processes, squark-squark pairs of the same charge should be selected. This can be achieved by studying like-sign di-lepton final states [5] in gaugino-type chargino and neutralino decay chains of two L- and R-type squarks like

$$
\begin{aligned}
\tilde{u}_{\mathrm{L}} & \rightarrow d+\tilde{\chi}_{1}^{+} \rightarrow \text { jet }+\ell^{+}+\not \\
\tilde{u}_{\mathrm{L}, \mathrm{R}} & \rightarrow u+\tilde{\chi}_{2}^{0} \rightarrow \text { jet }+\ell^{+} / \ell^{-}+\not \\
\tilde{d}_{\mathrm{L}} & \rightarrow u+\tilde{\chi}_{1}^{-} \rightarrow \text { jet }+\ell^{-}+\not \\
\tilde{d}_{\mathrm{L}, \mathrm{R}} & \rightarrow d+\tilde{\chi}_{2}^{0} \rightarrow \text { jet }+\ell^{-} / \ell^{+}+E
\end{aligned}
$$

with $\not E$ denoting the missing energy. SU(2) isospin invariance guarantees that the partial widths for $\tilde{u}_{\mathrm{L}}$ and $\tilde{d}_{\mathrm{L}}$ decays to charginos are equal to very good approximation in this configuration. Assuming CP-invariance, the subsequent leptonic decays of $\tilde{\chi}_{1}^{-}$are related to $\tilde{\chi}_{1}^{+}$, and the $\tilde{q}_{\mathrm{L}}^{*}$ decays to the $\tilde{q}_{\mathrm{L}}$ decays. For pure $\mathrm{SU}(2)$ singlet gaugino LSP states $\chi_{1}^{0}$, R-type squarks $\tilde{q}_{R}$ will decay into the channel $q_{R}+\chi_{1}^{0}$, not generating charged leptons in the final state. 

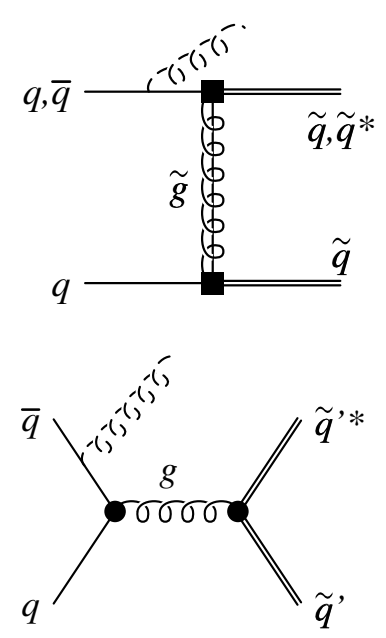
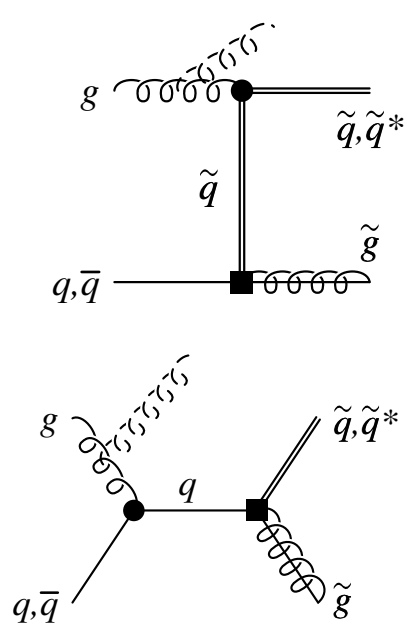
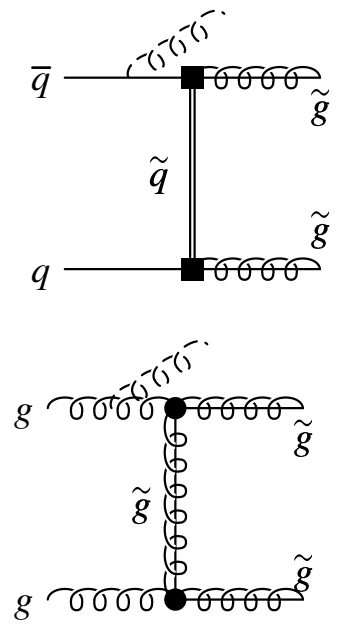

Figure 1: Characteristic examples of Feynman diagrams for partonic squark and gluino production in hadron collisions. Dots indicate the gauge coupling $g_{\mathrm{s}}$, while squares represent for the Yukawa coupling $\hat{g}_{\mathrm{s}}$. Gluon radiation in the initial state, indicated by the dashed lines, can lead to extra hard jets.

Extended mixing in the third-generation stop and sbottom sector will significantly increase the complexity of the analysis [though the production of stops may prove particularly useful in testing the Majorana character of gluinos [9]]. However, scalar top and bottom decays generate $b$ quarks in the final states which, by tagging, can be used to strongly reduce the influence of these sparticles on the analysis.

The branching ratios for gluino decays to squarks plus quarks depend only on the gluino, squark and quark masses so that they can be predicted unambiguously in the Compton process. To leading order the widths are given by the Yukawa couplings which however cancel from the branching ratios.

\section{Di-lepton plus jet analyses in the MSSM}

In the first step we have elaborated on the details of the scheme outlined above within the SUSY scenario defined in ref. [5]. Apart from the slightly increased gluino mass to improve on jet tagging, the scenario corresponds to the reference point SPS1a [10], describing a specific light-mass scenario within the minimal supersymmetric standard model MSSM. ${ }^{1}$ Typical parameters, masses and branching ratios relevant to the present analysis, are collected in table 1. This scenario should be interpreted as illustration for a large class of models characterized by the following two requirements: (i) To accumulate a sufficiently large event sample, the squark and gluino production cross sections must be large, and hence their masses correspondingly small. (ii) The gluino mass is heavier than the squark masses, with a gap sufficiently large to generate hard jets in gluino decays. At the time

\footnotetext{
${ }^{1}$ The model in ref. [5] is defined strictly at the weak scale and is not intended to accurately reflect either GUT scale physics or cosmological parameters which most likely are affected by other mechanisms too.
} 


\begin{tabular}{|rllllll|}
\hline \multicolumn{7}{c|}{ (a) Weak scale soft supersymmetry breaking parameters: } \\
$M_{1}=101 \mathrm{GeV}$ & $m_{\mathrm{L} 3}=199 \mathrm{GeV}$ & $m_{\mathrm{Q} 1}=551 \mathrm{GeV}$ & $m_{\mathrm{Q} 3}=499 \mathrm{GeV}$ \\
$M_{2}$ & $=192 \mathrm{GeV}$ & $m_{\mathrm{R} 3}=136 \mathrm{GeV}$ & $m_{\mathrm{U} 1}=529 \mathrm{GeV}$ & $m_{\mathrm{U} 3}=418 \mathrm{GeV}$ \\
$\mu$ & $=352 \mathrm{GeV}$ & & & $m_{\mathrm{D} 1}=526 \mathrm{GeV}$ & $m_{\mathrm{D} 3}=523 \mathrm{GeV}$ \\
$\tan \beta$ & $=10$ & & $A_{\tau}=-256 \mathrm{GeV}$ & $A_{\mathrm{b}}=-797 \mathrm{GeV}$ & $A_{\mathrm{b}}=-505 \mathrm{GeV}$
\end{tabular}

\begin{tabular}{|llllll|}
\hline \multicolumn{7}{c|}{ (b) Superpartner pole masses: } \\
$m_{\tilde{u}_{\mathrm{L}}}=567 \mathrm{GeV}$ & $m_{\tilde{d}_{\mathrm{L}}}=573 \mathrm{GeV}$ & $m_{\tilde{u}_{\mathrm{R}}}=547 \mathrm{GeV}$ & $m_{\tilde{d}_{\mathrm{R}}}=546 \mathrm{GeV}$ \\
$m_{\tilde{t}_{1}}=396 \mathrm{GeV}$ & $m_{\tilde{b}_{1}}=515 \mathrm{GeV}$ & $m_{\tilde{t}_{2}}=586 \mathrm{GeV}$ & $m_{\tilde{b}_{2}}=544 \mathrm{GeV}$ \\
$m_{\tilde{\chi}_{1}^{0}}=97 \mathrm{GeV}$ & $m_{\tilde{\chi}_{2}^{0}}=181 \mathrm{GeV}$ & $m_{\tilde{\chi}_{1}^{ \pm}}=176 \mathrm{GeV}$ & $m_{\tilde{\chi}_{3,4}^{0}}, m_{\tilde{\chi}_{2}^{ \pm}} \sim 370 \mathrm{GeV}$ \\
$m_{\tilde{\tau}_{1}}=136 \mathrm{GeV}$ & $m_{\tilde{\tau}_{2}}=208 \mathrm{GeV}$ & $m_{\tilde{g}}=700 \mathrm{GeV}$ &
\end{tabular}

\begin{tabular}{|lrlr|}
\hline & (c) Relevant branching ratios: \\
$\operatorname{BR}\left[\tilde{u}_{\mathrm{L}} \rightarrow d \tilde{\chi}_{1}^{+}\right]=65 \%$ & $\operatorname{BR}\left[\tilde{\chi}_{1}^{ \pm} \rightarrow \tilde{\tau}^{ \pm} \nu_{\tau}\right]=95 \%$ & $\operatorname{BR}\left[\tilde{g} \rightarrow q \tilde{q}_{\mathrm{L}}\right]=5.5 \%, \quad q \neq b, t$ \\
$\operatorname{BR}\left[\tilde{d}_{\mathrm{L}} \rightarrow u \tilde{\chi}_{1}^{-}\right]=61 \%$ & $\operatorname{BR}\left[\tilde{\chi}_{1}^{ \pm} \rightarrow W^{* \pm} \tilde{\chi}_{1}^{0}\right]=5 \%$ & $\operatorname{BR}\left[\tilde{g} \rightarrow q \tilde{q}_{\mathrm{R}}\right]=7.3 \%$, & $q \neq b, t$ \\
$\operatorname{BR}\left[\tilde{u}_{\mathrm{L}} \rightarrow u \tilde{\chi}_{2}^{0}\right]=32 \%$ & & $\operatorname{BR}\left[\tilde{g} \rightarrow b \tilde{b}_{i}\right]=17.5 \%$ \\
$\operatorname{BR}\left[\tilde{d}_{\mathrm{L}} \rightarrow d \tilde{\chi}_{2}^{0}\right]=31 \%$ & $\operatorname{BR}\left[\tilde{g} \rightarrow b \tilde{t}_{i}\right]=31 \%$ \\
\hline
\end{tabular}

Table 1: Reference scenario used in this analysis. It coincides with the MSSM Snowmass point SPS1a [10], except for the slightly larger gluino mass [5]. The low-energy spectrum has been calculated by means of SoftSUSY 1.9.1 [1].

when the experimental analysis would be carried out, the relevant properties of the spectrum will have been determined already, and hence the analysis can be tailored accordingly. If the conditions which this study is based upon are not met, the methods would need to be adjusted, e.g. by analyses of cascade decays for reverse gluino/squark mass ratios.

Similarly to ref. [5], we will assume in this first step that all the parameters, masses and branching ratios, have been pre-determined in squark cascades at LHC and/or analyses at an $e^{+} e^{-}$linear collider. It should be noted, however, that within the group-theoretical frame of the minimal supersymmetric extension of the Standard Model [MSSM], the squark branching ratios can be predicted if the structure of the light chargino/neutralino sector has been explored at a low-energy lepton collider. Within this framework the measurement of the squark-decay branching ratios, experimentally difficult to access, can be substituted by the completeness assumption of the MSSM. The assumption can be cross-checked internally at LHC by searching for non-MSSM squark decays.

About two thirds of the time, the light-flavor L-squarks decay into the charginos $\tilde{\chi}_{1}^{ \pm}$, while about one third of the decays go into the neutralino $\tilde{\chi}_{2}^{0}$, following from the fact that the L-squarks have small hypercharges, $Y_{\tilde{q}_{\mathrm{L}}}=1 / 6$, and thus predominantly couple to winos. Furthermore, since the mixing between the bino and wino components of the neutralinos is small, as well as the mixing between L- and R-squark states negligible, the branching fractions of the up- and down-squarks are almost equal. This is in particular the case in our reference scenario, $c f$. table 1, and a consequence of $\mathrm{SU}(2)$ and $\mathrm{CP}$ invariance. [Note 
that in general CP invariance can be broken in the MSSM by complex parameters, but in this analysis all supersymmetry and supersymmetry breaking parameters are assumed to be real.]

Due to the relatively large value of $\tan \beta=10$ in this scenario, the charginos $\tilde{\chi}_{1}^{ \pm}$ almost exclusively decay to the scalar tau $\tilde{\tau}_{1}$, which subsequently decays to taus. To trace the charge of the squarks explicitly, we restrict ourselves to the leptonic tau decays. The phenomenological analysis we will therefore be designed such that the decay chains

$$
\begin{aligned}
& \tilde{u}_{\mathrm{L}} \stackrel{65 \%}{\longrightarrow} d \tilde{\chi}_{1}^{+} \stackrel{100 \%}{\longrightarrow} d \tau^{+} \nu_{\tau} \tilde{\chi}_{1}^{0} \stackrel{35 \%}{\longrightarrow} d \ell^{+}+\not \\
& \tilde{d}_{\mathrm{L}} \stackrel{61 \%}{\longrightarrow} u \tilde{\chi}_{1}^{-} \stackrel{100 \%}{\longrightarrow} u \tau^{-} \bar{\nu}_{\tau} \tilde{\chi}_{1}^{0} \stackrel{35 \%}{\longrightarrow} u \ell^{-}+\not E[\ell=e, \mu]
\end{aligned}
$$

are singled out. The numbers above the arrows denote the branching fractions.

The LHC processes which have been studied in this report are like-sign di-lepton final states plus a number of jets with variable transverse momenta:

$$
\begin{aligned}
& p p \rightarrow \ell^{ \pm} \ell^{ \pm}+2 \text { jets }+E \\
& p p \rightarrow \ell^{ \pm} \ell^{ \pm}+3 \text { jets }+E \\
& p p \rightarrow \ell^{ \pm} \ell^{ \pm}+4 \text { jets }+E
\end{aligned}
$$

and possibly additional jets from gluon radiation, that however tend to have smaller transverse energies than the jets emitted in squark and gluino decays.

The first process, eq. (3.2), concentrates on direct squark-pair production, the classical reaction [5] for measurements of the strong Yukawa coupling with the basic cross section scaling as $\hat{g}_{\mathrm{s}}^{4}$. The transverse momentum of the jets is assumed beyond $200 \mathrm{GeV}$.

In the second process, eq. (3.3), we demand two high transverse momentum jets with $E_{\mathrm{T}} \geq 200 \mathrm{GeV}$, plus an additional third jet with variable transverse momentum. For low transverse momenta of the third jet, direct squark-pair production is projected out, moving the dominant weight to the Compton process however for increasing transverse momentum. In this way, depending on the transverse momentum, different powers of the Yukawa coupling, $\sim c_{1}\left(p_{\mathrm{T}}\right) \hat{g}_{\mathrm{s}}^{4}+c_{2}\left(p_{\mathrm{T}}\right) g_{\mathrm{s}}^{2} \hat{g}_{\mathrm{s}}^{2}$, become relevant.

The evaluation of the third process, eq. (3.4), parallels the previous analysis. It does not provide new methodological insight but increases statistics when included.

To analyse the prospects for extracting the SUSY-QCD Yukawa coupling in a realistic experimental environment, we have generated samples for the squark and gluino production process as well as SM backgrounds with PyтніA 6.408 [12], including gluon and quark radiation through virtuality-ordered parton showers, string hadronisation and the underlying event according to 'Tune A' 13]. The required modifications for varying the SUSY-QCD Yukawa coupling independently of the gauge coupling were implemented in a private version, ${ }^{2}$ according to the lowest-order cross sections given in [6]. The main SM backgrounds originate from $t \bar{t}$ production as well as $W W j j$ production. The $W W j j$ process was generated with MADGRAPH [14], without QCD radiation and fragmentation.

\footnotetext{
${ }^{2}$ To be made publicly available from PYTHIA version 6.411 .
} 
The cross-sections for squark, gluino and top production were normalised by $K$-factors to the values obtained with next-to-leading order QCD corrections [6, 15], while for the $W^{ \pm} W^{ \pm} j j$ background only leading order results are available. In analysing distributions, $\mathrm{K}$-factors as calculated for the total cross-sections have been included; this approximation is based on conclusions of ref. [6] where the primary distributions have been shown not to be distorted by more than $10 \%$ so that a uniform rescaling is justified. A possible complication is created by the fact that the gluino is quite wide in our scenario, with $\Gamma_{\tilde{g}} \sim$ $35 \mathrm{GeV}$ at leading order, generating doubts whether the narrow-width limit is justified. For example, the convolution of the corresponding Breit-Wigner function(s) with the steeply falling parton distributions greatly distorts the final distribution — to the point of even creating a spurious peaking towards low masses. We note that this issue is generic for gluon-induced processes involving wide resonances at the LHC, and although we do not aim to solve it here, the reader should be aware of this problem.

Finally, the detector response was parametrised by simple acceptance cuts: a toy calorimeter spanning the pseudorapidity range $|\eta|<5$ with a resolution of $0.1 \times 0.1$ in $\eta \times \phi$ space was assumed. Jets were identified with a simple UA1-like cone jet algorithm (PYCELL), with a cone size of $\Delta R=0.4$. Muons and electrons were reconstructed inside $|\eta|<2.5$ and an isolation criterion was further imposed, requiring both less than $10 \mathrm{GeV}$ of additional energy deposited in a cone of size $\Delta R=0.2$ around the lepton and also no reconstructed jets with $p_{\mathrm{T}, \mathrm{j}}>25 \mathrm{GeV}$ closer than $\Delta R=0.4$ around the lepton. These criteria duplicate the default settings of the ATLFAST simulation package [16].

All the signal processes, eqs. (3.2) to (3.4), are characterised by the similar features of two leptons, missing energy and at least two hard jets. Therefore the following preselection cuts have been used in the event generation:

- At least $E>100 \mathrm{GeV}$ of missing energy.

- At least 2 jets with $p_{\mathrm{T}, \mathrm{j}}>100 \mathrm{GeV}$.

- Exactly two isolated same-sign leptons $\ell=e, \mu$ with $p_{\mathrm{T}, \ell}>7 \mathrm{GeV}$.

To reduce the SM background, the final analysis in addition employs the soft selection cuts

- At least $E>300 \mathrm{GeV}$ of missing energy.

- At least 2 jets with $p_{\mathrm{T}, \mathrm{j}}>200 \mathrm{GeV}$.

- Veto on identified bottom-quark jets, using a tagging efficiency of $\epsilon=90 \%$ with a mistagging rate $D=25 \%$ [16, 5].

These cuts have been optimised to minimise the remaining SM background, however at the cost of being relatively expensive on the signal statistics as well. This choice is justified by the goal to interpret the measured signal rate and distributions as a combination of $\tilde{u}, \tilde{d}$ squark and $\tilde{g}$ gluino production processes, so that it is mandatory to keep the contamination from background processes as low as possible.

The veto on bottom quarks is effective against the $t \bar{t}$ background, but it also helps to reject the unwanted decay modes of the gluinos into scalar bottoms and scalar tops. Since 


\begin{tabular}{|l|r|r|r|}
\hline Process & Total cross-section & Cross-section with BRs and cuts & Efficiency \\
\hline$\sigma\left[\tilde{q}_{\mathrm{L}} \tilde{q}_{\mathrm{L}}\right]$ & $2.1 \mathrm{pb}$ & $6.1 \mathrm{fb}$ & $0.29 \%$ \\
$\sigma\left[\tilde{q}_{\mathrm{L}} \tilde{q}_{\mathrm{L}}^{*}\right]$ & $1.4 \mathrm{pb}$ & $3.1 \mathrm{fb}$ & $0.23 \%$ \\
$\sigma\left[\tilde{q}_{\mathrm{L}} \tilde{g}\right]$ & $7.0 \mathrm{pb}$ & $5.8 \mathrm{fb}$ & $0.08 \%$ \\
$\sigma[\tilde{g} \tilde{g}]$ & $3.2 \mathrm{pb}$ & $0.8 \mathrm{fb}$ & $0.03 \%$ \\
\hline$\sigma[\mathrm{SM}]$ & $800 \mathrm{pb}$ & $<0.6 \mathrm{fb}$ & \\
\hline
\end{tabular}

Table 2: Signal and background cross-sections after applying the cuts mentioned in the text.

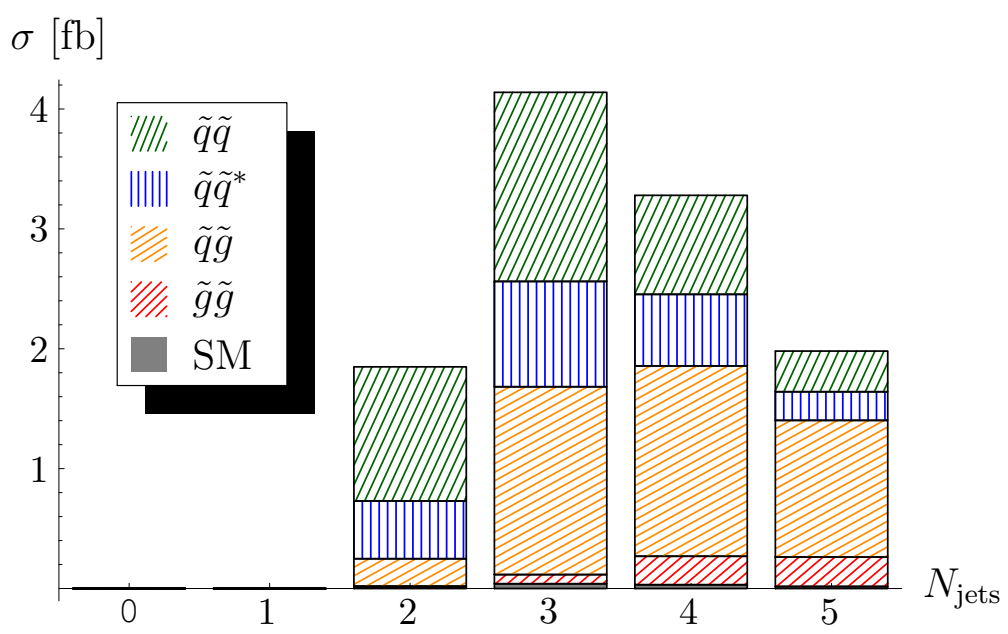

Figure 2: Results of simulations for the number of identifiable jets, for the different processes that lead to the general signatures in eqs. (3.2) to (3.4). For the first two jets, a threshold of $p_{\mathrm{T}, \text { jet }}>200 \mathrm{GeV}$ has been used, while $p_{\mathrm{T}, \text { jet }}>50 \mathrm{GeV}$ for the other jets.

the stop and sbottom decay chains can deviate significantly from those of the light-flavour squarks, it is advantageous to remove them from the analysis. As mentioned above, the relevant branching ratios of the gluinos into different squark flavours can be determined unambiguously from the gluino, squark and quark masses.

After application of the cuts, the signal and background cross-sections listed in table 2 are obtained. Thus, with a total luminosity of $300 \mathrm{fb}^{-1}$ about 2700 squark pair events and 1750 events from the Compton process are expected.

After reducing the SM background, the three high-energy processes $p p \rightarrow \tilde{q}_{\mathrm{L}} \tilde{q}_{\mathrm{L}}, \tilde{q}_{\mathrm{L}} \tilde{g}$, $\tilde{g} \tilde{g}$ should be distinguished in order to extract the SUSY-QCD Yukawa coupling $\hat{g}_{\mathrm{s}}$. As outlined above, in a first step this can be achieved, statistically, by analysing the number of hard jets in the final state, see figure 2. With a transverse momentum threshold of $p_{\mathrm{T}, \text { jet }}>50 \mathrm{GeV}$ for the jets beyond the two hardest jets (with $p_{\mathrm{T} \text {,jet }}>200 \mathrm{GeV}$ ), the sample of events with only two jets is dominated by squark pair production. On the other hand, events with three or more jets largely originate from the Compton process $p p \rightarrow \tilde{q}_{\mathrm{L}} \tilde{g}$. 
For $N_{\text {jets }} \geq 4$, gluino pair production also contributes to the di-lepton + jets signal, though the rate for this process is strongly suppressed due to the $b$ veto.

From the measured rates for events with 2, 3 and 4 jets, and using the pre-determined branching ratios for squarks and gluinos, the coupling $\hat{g}_{\mathrm{s}}$ can be determined. A similar analysis has already been performed in ref. [5], but only using the 2-jet portion. Including the higher statistics of the 3 - and 4-jet events, we conclude that a much more precise measurement of $\hat{g}_{\mathrm{s}}$ could be possible. From a fit to the simulated data, the statistical error is estimated to be only $0.4 \%$, assuming a total luminosity of $300 \mathrm{fb}^{-1}$.

With this precision, systematic errors might become the limiting factor for the accurate determination of $\hat{g}_{\mathrm{s}}$. We have identified and estimated the following dominant sources of systematic uncertainties.

The extraction of a cross section for a hard production process at a hadron collider depends on the knowledge of the parton distribution functions (PDFs) of the protons in the initial state. It is likely that our knowledge of PDFs for processes with large transverse momentum will be improved by data taken at the LHC itself. Nevertheless, here we adopt a conservative approach and estimate the uncertainty resulting from PDFs by comparing results for different CTEQ PDFs releases (CTEQ6M, CTEQ6D, CTEQ5M1, CTEQ5L and CTEQ6L1) [17 that are available today. It is found that this variation leads to an uncertainty of about $1 \%$ in the extracted Yukawa coupling.

Furthermore, the precise analysis of the squark and gluino cross sections relies on accurate squark and gluino mass measurements and on theoretical calculations of higherorder corrections. For the sparticle mass errors, we have adopted the results of ref. [19], where an uncertainty of about $10 \mathrm{GeV}$ is expected for the L-squark masses and about $12 \mathrm{GeV}$ for the gluino masses. On the theoretical side, the next-to-leading order corrections are known [6] and have been included in the analysis. The uncertainty of the missing $\mathcal{O}\left(\alpha_{\mathrm{s}}^{2}\right)$ contributions are estimated by varying the renormalisation scale of the $\mathcal{O}\left(\alpha_{\mathrm{s}}\right)$ corrected cross section between $m / 2<Q<2 m$, where $m$ is the average mass of the produced sparticles (squark or gluino).

The error estimate does not include the uncertainty for the squark branching ratios which are presumed to be small. In practice, due to the large squark masses in our reference scenario, they could either be measured at an $e^{+} e^{-}$linear collider with energy beyond $1 \mathrm{TeV}$ or, based alternatively on the closure of the MSSM, one could reconstruct the neutralino/chargino sector at a $500 \mathrm{GeV}$ linear collider with high precision at the per-cent to per-mil level [3, 18] and derive the squark branching fractions from this information.

The effect of the systematic errors and the combined error, obtained by adding all individual error sources in quadrature, are listed in table 3 (Method A). It turns out that the total error, $\delta\left[\hat{g}_{\mathrm{s}} / g_{\mathrm{s}}\right]=3.4 \%$ is dominated by systematic effects.

With a luminosity upgrade of the LHC, yielding a total luminosity of $1 \mathrm{ab}^{-1}$, and assuming that the mass and PDF uncertainties will improve with higher luminosity, this error could be reduced to $2.5 \%$. 


\begin{tabular}{|c|c|c|c|}
\hline Error source & \multicolumn{3}{|c|}{$\delta\left[\hat{g}_{\mathrm{s}} / g_{\mathrm{s}}\right]$} \\
\hline Method & A & B & $\mathrm{C}$ \\
\hline LHC statistics for $300 \mathrm{fb}^{-1}$ & $0.6 \%$ & $3.3 \%$ & $5.7 \%$ \\
\hline PDF uncertainty & $1.4 \%$ & $2.9 \%$ & $2.9 \%$ \\
\hline NNLO corrections & $2.0 \%$ & $3.1 \%$ & $3.1 \%$ \\
\hline Mass measurements $\Delta m_{\tilde{q}}=10 \mathrm{GeV}$ & $2.0 \%$ & $1.1 \%$ & $1.1 \%$ \\
\hline$\Delta m_{\tilde{g}}=12 \mathrm{GeV}$ & $1.0 \%$ & $2.0 \%$ & $2.0 \%$ \\
\hline Total & $3.4 \%$ & $5.9 \%$ & $7.5 \%$ \\
\hline
\end{tabular}

Table 3: Combination of statistical and systematic errors for extracting the SUSY-QCD Yukawa coupling from like-sign dilepton events including jet transverse-momentum distributions at the LHC. In Method A the complete information on squark branching ratios from a multi-TeV $e^{+} e^{-}$ collider is used for an SPS1a-type scenario. Method B is based on LHC data potentially alone, but supported eventually by $1 \mathrm{TeV} e^{+} e^{-}$collider data, and combined with moderate assumptions on new heavy iso-scalar singlets. Method $\mathrm{C}$ allows for more complex scenarios in the neutralino sector than encoded in SPS1a-type reference points, allowing again for the presence of new Standard Model singlets.

\section{Towards model-independent analyses}

The procedure of determining the Yukawa coupling $\hat{g}_{\mathrm{s}}$ as outlined in the previous section can be expanded towards a more general analysis. Though the problem is too complex to allow for a completely model-independent analysis, methods can nevertheless be exploited which are based only on the pre-determined chargino/neutralino system, as expected to be known from LHC and/or $e^{+} e^{-}$collider measurements, combined with natural assumptions on the structure of theories beyond the minimal supersymmetric extension of the Standard Model MSSM. Central to this approach is the circumvention of external measurements of heavy squark-decay branching ratios which, for general expectations of the spectrum, can only be performed at a multi-TeV lepton collider.

\subsection{MSSM plus heavy iso-singlets}

First we will analyse a scenario in which the MSSM is expanded by a set of heavy isoscalar particles, with little mixing between standard and new states, as could be expected in extended Higgs and gauge theories, see e.g. refs. [20].

Proceeding from the same scenario as analysed in section 3, we observe that the charginos and neutralinos are light enough to be produced as diagonal and mixed pairs in $e^{+} e^{-}$collisions below $1 \mathrm{TeV}, \tilde{\chi}_{i}^{+} \tilde{\chi}_{j}^{-}(i, j=1,2)$ and $\tilde{\chi}_{i}^{0} \tilde{\chi}_{j}^{0}(i, j=1, \ldots, 4)$. Masses and mixing parameters can be measured in $e^{+} e^{-}$experiments, $c f$. refs. [3], possibly even by observing only a subgroup of the lightest particles 21]. They determine the SU(2), U(1) gaugino and higgsino decomposition of the charginos and neutralinos. Thus the partial widths $\Gamma(\tilde{q} \rightarrow \tilde{\chi})$ can be predicted from these experimental results. Moreover, the decay 
branching ratios $B R\left(\tilde{\chi} \rightarrow \ell^{ \pm}\right)$can be measured directly. In many scenarios even the observation of relatively large mass differences among the neutralinos and charginos [possible at LHC already] is sufficient to suggest strongly that the mixing in the neutralino and chargino sector is very small and thus can be neglected for the purposes here. This is the case, for instance, in the reference scenario studied in this paper.

While the partial widths $\Gamma(\tilde{q} \rightarrow \tilde{\chi} \rightarrow \ell)$ can be completely determined in this scenario, the total widths $\Gamma(\tilde{q})$, and as a result the branching ratios, remain unknown since decay channels to heavy particles beyond MSSM, in particular to heavy iso-singlets, with unknown decay characteristics, may be open. However, since R-squarks do not decay to leptons in scenarios where the lightest neutralino is predominantly bino, the only unknown quantities are the branching ratios $B R\left(\tilde{u}_{L} \rightarrow \tilde{\chi}^{+}\right)$and $B R\left(\tilde{d}_{L} \rightarrow \tilde{\chi}^{-}\right)$to which all other branching fractions can be linked once the $\tilde{\chi}$ mixing matrices are known; according to the WignerEckart theorem:

$$
\begin{aligned}
& B R\left(\tilde{u}_{L} \rightarrow \tilde{\chi}_{2}^{0}\right)=\frac{1}{2} f_{u u}\left(N^{ \pm}, N^{0}\right) B R\left(\tilde{u}_{L} \rightarrow \tilde{\chi}_{1}^{+}\right) \\
& B R\left(\tilde{d}_{L} \rightarrow \tilde{\chi}_{2}^{0}\right)=\frac{1}{2} f_{d d}\left(N^{ \pm}, N^{0}\right) B R\left(\tilde{d}_{L} \rightarrow \tilde{\chi}_{1}^{-}\right)
\end{aligned}
$$

The coefficients $f_{u u, d d}$ are ratios of the chargino $N^{ \pm}$and the neutralino $N^{0}$ mixing matrix elements, with $\frac{1}{2}$ being the ratio of the standard Clebsch-Gordan coefficients squared. For pure $\tilde{\chi}_{1}^{ \pm}, \tilde{\chi}_{2}^{0}$ iso-vector winos, the relations simplify to $f_{u u, d d} \rightarrow 1$.

This could be the platform for a 3-parameter analysis, including the unknown branching ratios and the strong Yukawa coupling. However, the two branching ratios above are related if squark decays beyond $\tilde{\chi}_{i}^{ \pm}$and $\tilde{\chi}_{i}^{0}$ involve only new iso-singlet particles with universal couplings to L-squarks, as may be expected, on quite natural grounds, outside the Standard Model. In the same notation as above, the relation reads

$$
B R\left(\tilde{d}_{L} \rightarrow \tilde{\chi}_{1}^{-}\right)=f_{d u}\left(N^{ \pm}, N^{0}\right) B R\left(\tilde{u}_{L} \rightarrow \tilde{\chi}_{1}^{+}\right)
$$

[with $f_{d u} \rightarrow 1$ in the wino limit]. In this scenario the analysis of section 3 repeats itself except for leaving free the overall normalisation, governed by the unknown branching ratio $B R\left(\tilde{u}_{L} \rightarrow \tilde{\chi}_{1}^{+}\right)$. In this case, the SUSY-QCD Yukawa coupling can only be extracted from ratios of the cross sections or from distributions with varying transverse momenta, but not from absolute values of cross sections.

By analysing the measured rates for events with 2, 3 and 4 jets, one can extract a ratio of the cross-sections for $\tilde{q}_{\mathrm{L}} \tilde{q}_{\mathrm{L}}$ and $\tilde{q}_{\mathrm{L}} \tilde{g}$ production. This has also been exploited for the model analyses in ref. [7]. By comparison with the theoretical calculations for these cross-sections, this ratio can then be interpreted in terms of the coupling ratio $\hat{g}_{\mathrm{s}} / g_{\mathrm{s}}$.

The robustness and precision of the Yukawa coupling determination can be improved by looking not only at the number of identified jets, but also their transverse momentum distribution.

The results of the simulations are presented in figures 3. As apparent from the figure, both the distributions as a function of the $p_{\mathrm{T}}$ of the third and the fourth jet can discriminate between the two processes eqs. (2.1) and (2.2). Squark-pair production tends to peak 


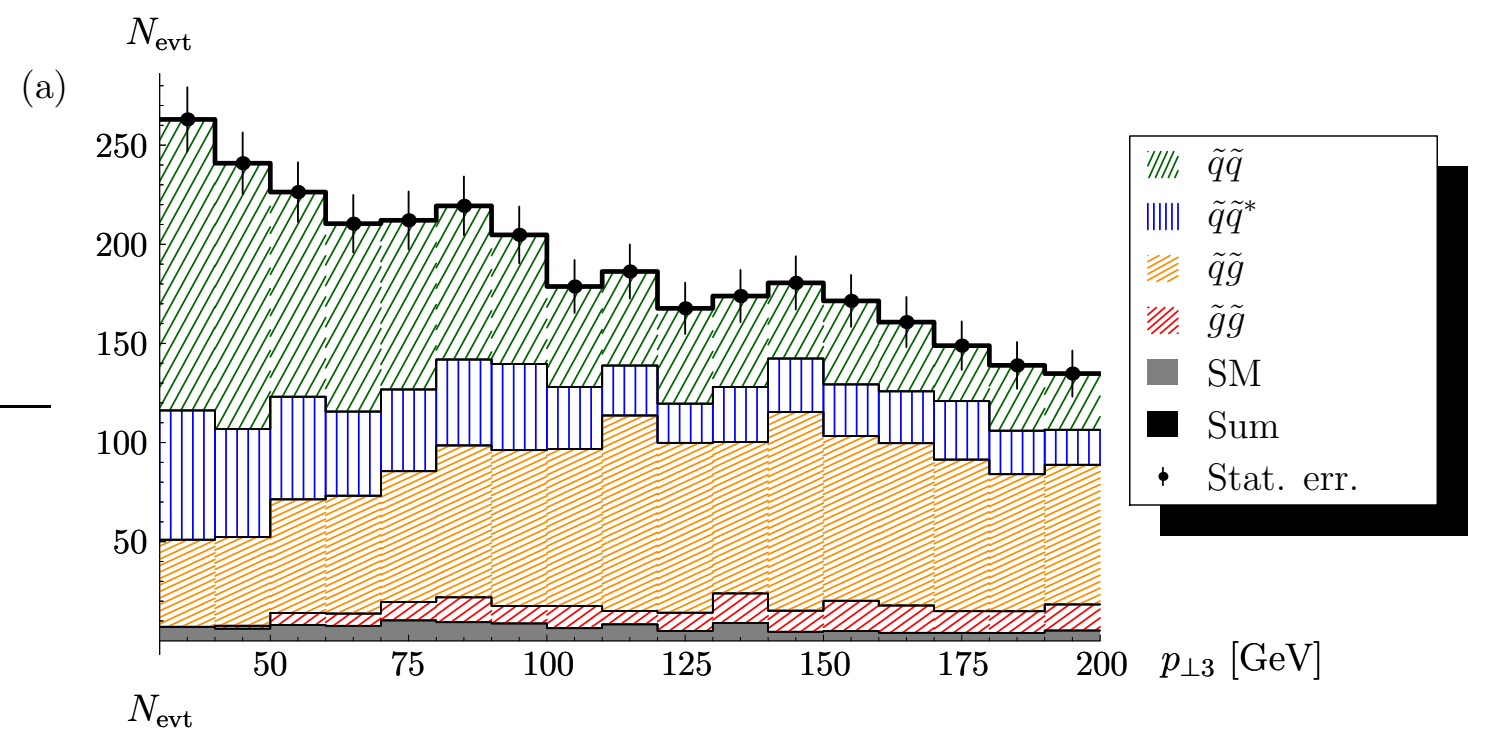

(b)

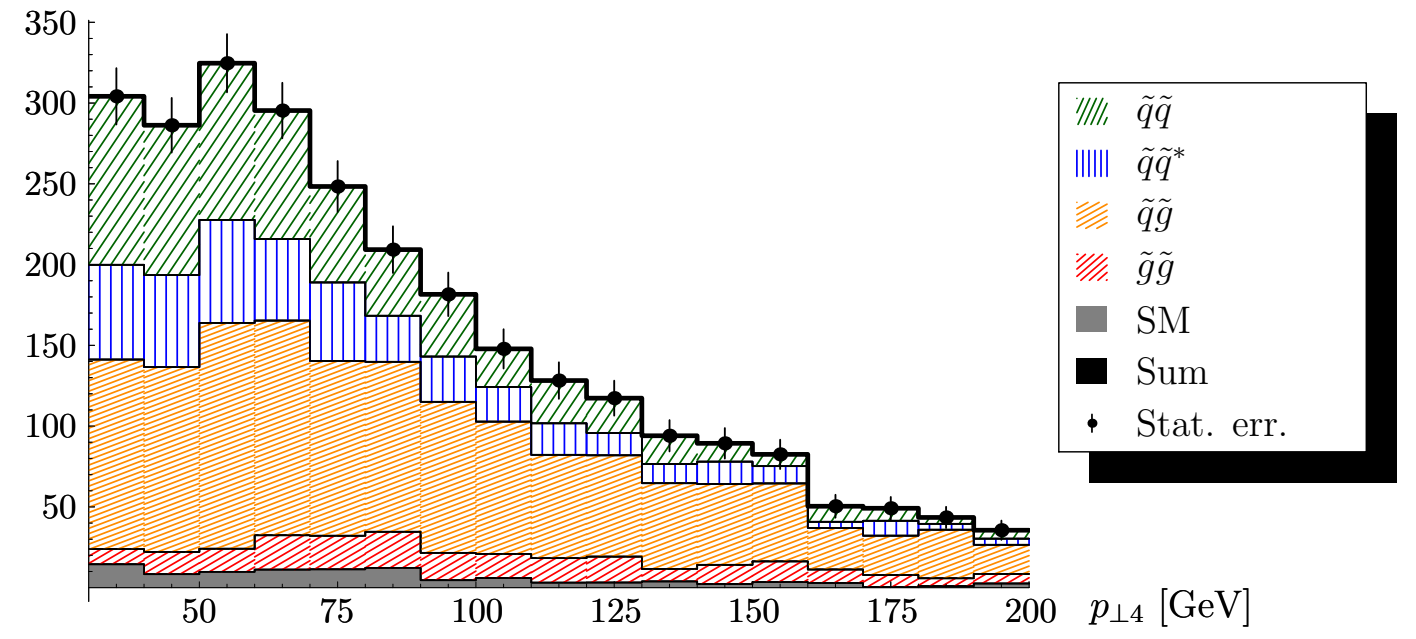

Figure 3: Results of simulations for (a) the distribution with respect to the $p_{\mathrm{T}}$ of the third jet, and (b) the distribution with respect to the $p_{\mathrm{T}}$ of the fourth jet (with $p_{\mathrm{T}, 3}>50 \mathrm{GeV}$ ). In both plots the contributions from the processes in eqs. (3.2) to (3.4) as well as the SM background are shown separately, while for the sum the statistical errors for $300 \mathrm{fb}^{-1}$ is also shown.

towards low values of $p_{\mathrm{T}, 3}$ and $p_{\mathrm{T}, 4}$, since the additional jets are generated in this process only by gluon radiation from the initial or final state. The $\tilde{q} \tilde{g}$ Compton production and the $\tilde{g} \tilde{g}$ gluino-pair production, on the other hand, typically lead to larger values for $p_{\mathrm{T}, 3}$ and $p_{\mathrm{T}, 4}$. Therefore, the lower ends of the $p_{\mathrm{T}, 3}$ or $p_{\mathrm{T}, 4}$ spectra depend more strongly on variations of $\hat{g}_{\mathrm{s}}$ than the region of higher transverse momenta. As a consequence, the shape of the transverse momentum distributions is sensitive to the ratio $\hat{g}_{\mathrm{s}} / g_{\mathrm{s}}$, independent of the total normalisation of the cross-section.

The expected experimental precision for the determination of the SUSY-QCD Yukawa coupling can be estimated from a binned $\chi^{2}$ fit. Assuming $300 \mathrm{fb}^{-1}$ of integrated luminosity, the statistical error for $\hat{g}_{\mathrm{s}} / g_{\mathrm{s}}$ is $3.3 \%$. This has to be combined with systematic errors as 


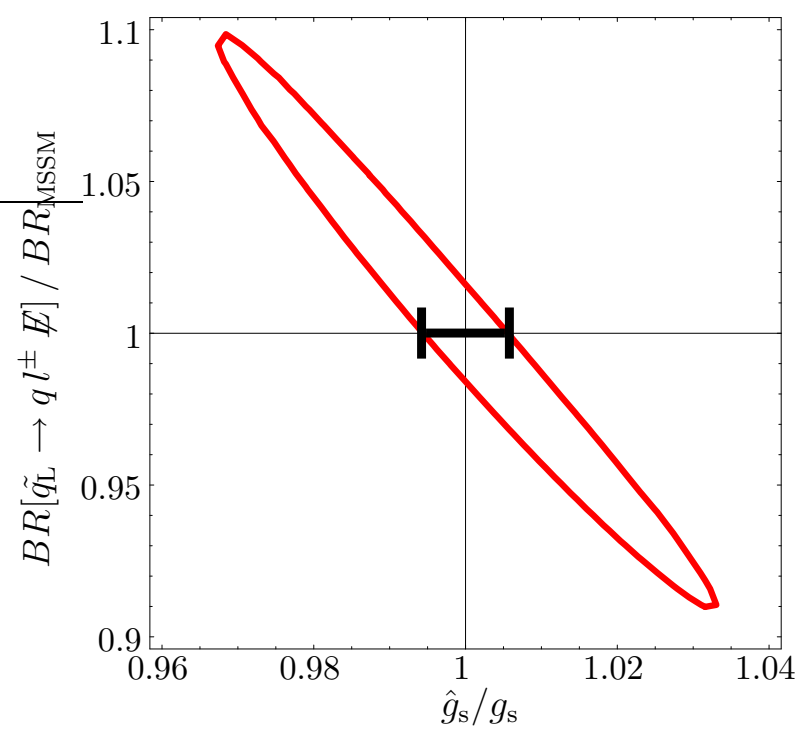

Figure 4: Independent results for the SUSY-QCD Yukawa coupling $\hat{g}_{\mathrm{s}}$ and the overall branching ratio of L-squarks into a single identified lepton $l=e, \mu$ (normalised to the MSSM value) from a fit to the $p_{\mathrm{T} 3}$ distribution in figure 3 (a). The plot shows the $1 \sigma$ statistical error contours corresponding to the fit method B. The black bar indicates the result from the fit method A, assuming that the branching ratio has been pre-determined.

given in table 3 (Method B), generating a final error of 5.9\%. The systematic errors were estimated in the same way as explained in the previous section.

Since the overall normalisation is left as a free parameter in this analysis, the result can also be exploited to determine the branching ratio of squark decays to charginos. A fit to $p_{\mathrm{T}, 3}$, for instance, can then simultaneously be interpreted as information about the Yukawa coupling and the squark branching ratio, see figure 4 .

\subsection{Generalised picture}

In the second more general step we drop the assumption that $\tilde{\chi}_{2}^{0}$ is nearly wino-like but allow, as before, for a set of new heavy singlets under the Standard Model group.

In this scenario, the neutralino sector becomes more involved. Significant mixing between bino and wino components in the neutralino $\tilde{\chi}_{2}^{0}$, for instance, can create a sizable branching ratio of R-squarks $\tilde{u}_{R}, \tilde{d}_{R}$ into leptons [considering, as before, the decay $\tilde{\chi}_{2}^{0} \rightarrow \tau^{+} \tau^{-} \tilde{\chi}_{1}^{0}$, with one tau decaying leptonically, the other hadronically, leads to one identified lepton in the final state]. Furthermore, mixing remnants with the extra isosinglet components eventually may make the picture even more complicated.

However, it should be noted that neutralinos always generate the same number of positively and negatively charged leptons. On the other hand, chargino decay chains lead to leptons in the final state whose charge is correlated to the squarks from which the charginos originate. Therefore, this scenario can still be controlled by measuring separately the cross sections for $\ell^{+} \ell^{+}$and $\ell^{-} \ell^{-}$production. All neutralino decay modes of $\mathrm{L}-$ and 
R-squarks can be combined into one contribution, leading to equal rates of $\ell^{+}$and $\ell^{-}$in the final state signature. Then, two branching ratios remain undetermined, $B R\left(\tilde{u}_{L} \rightarrow \tilde{\chi}_{1}^{+}\right)$ and $B R\left(\tilde{d}_{L} \rightarrow \tilde{\chi}_{1}^{-}\right)$, that control the difference between the rates for $\ell^{+} \ell^{+}$and $\ell^{-} \ell^{-}$.

This analysis relies mainly on the assumption that the chargino sector remains unmodified with respect to the MSSM when the system is expanded. [Only additional new charginos that couple differently to $\tilde{u}_{L}$ and $\tilde{d}_{L}$ would significantly alter that picture.] In any case, the chargino sector can be brought under control from measurements at a lowenergy lepton collider. Furthermore, for our analysis of the signature of two leptons, two or more jets and missing energy, we will obtain a decent signal only for the mass hierarchy $m_{\tilde{g}} \gg m_{\tilde{q}} \gg m_{\tilde{\chi}_{1}^{ \pm}} \gg m_{\tilde{\chi}_{1}^{0}}$. For different mass spectra, the selection cuts would effectively kill the SUSY signal. It is likely that a similar analysis can be performed in such a case by suitably adjusting the cuts, but a thorough investigation of all possible situations is beyond the scope of this paper.

We repeat the analysis of the previous section, now taking as measurable observables the [binned] $p_{\mathrm{T}}$ spectra of the $3 \mathrm{rd}$ and 4 th jet for $\ell^{+} \ell^{+}$and $\ell^{-} \ell^{-}$separately. The free parameters in the fit are the Yukawa coupling $\hat{g}_{\mathrm{s}}$ and the branching fractions $B R\left(\tilde{u}_{L} \rightarrow \tilde{\chi}_{1}^{+}\right)$ and $B R\left(\tilde{d}_{L} \rightarrow \tilde{\chi}_{1}^{-}\right)$, in addition the overall normalisation and the contribution from Rsquark processes, mainly $p p \rightarrow \tilde{q}_{\mathrm{L}}^{(*)} \tilde{q}_{\mathrm{R}}^{(*)}$. [On the methodological side, this case illustrates most clearly the technical potential of varying the relative weight of direct squark-pair production versus the Compton process in determining the Yukawa coupling.] Since the closure of the minimal supersymmetric Standard Model, in the form defined above, is a natural assumption, the scenario is expected to be quite general and comprehensive. Weighing this qualification properly, the separate $\ell^{+} \ell^{+}, \ell^{-} \ell^{-}$measurements come very close to a model-independent determination of the Yukawa coupling $\hat{g}_{\mathrm{s}}$.

Assuming a luminosity of $300 \mathrm{fb}^{-1}$, a binned $\chi^{2}$ fit gives a statistical error of $5.7 \%$ for $\hat{g}_{\mathrm{s}}$, see table 3 (Method C). The individual squark branching ratios, on the other hand, are determined relatively poorly, see figure 5 . The origin of this uncertainty is the fact that both the $p_{\mathrm{T}}$ spectra as well as the ratio of $\left(\ell^{+} \ell^{+}\right) /\left(\ell^{-} \ell^{-}\right)$signal rates discriminate a priori hardly between $\tilde{u}_{\mathrm{L}}$ and $\tilde{d}_{\mathrm{L}}$ decays. The distinction is slightly enhanced by the different shapes of $u$ - and $d$-quark distributions in the proton, though the fit results for $B R\left(\tilde{u}_{L} \rightarrow \tilde{\chi}_{1}^{+}\right)$and $B R\left(\tilde{d}_{L} \rightarrow \tilde{\chi}_{1}^{-}\right)$remain strongly correlated. The correlation however does not affect the accuracy of the Yukawa coupling significantly. With a relative contribution of $12 \%$ to ++ and $20 \%$ to -- final states, respectively, $\tilde{\chi}_{2}^{0}$ pairs contribute moderately to the signal events, but they lead to almost the same transverse jet momentum distribution as the chargino channels. This explains the relatively precise determination of $\hat{g}_{\mathrm{s}}$, even when the squark branching fractions are not pre-determined.

The systematic errors in the analysis method $\mathrm{C}$ are equivalent to Method B. In total, the error on the ratio $\hat{g}_{\mathrm{s}} / g_{\mathrm{s}}$ can be expected close to $7.5 \%$ in this largely model-independent approach. With a luminosity-upgraded LHC, yielding $1000 \mathrm{fb}^{-1}$, this error could be reduced to about $5 \%$. 

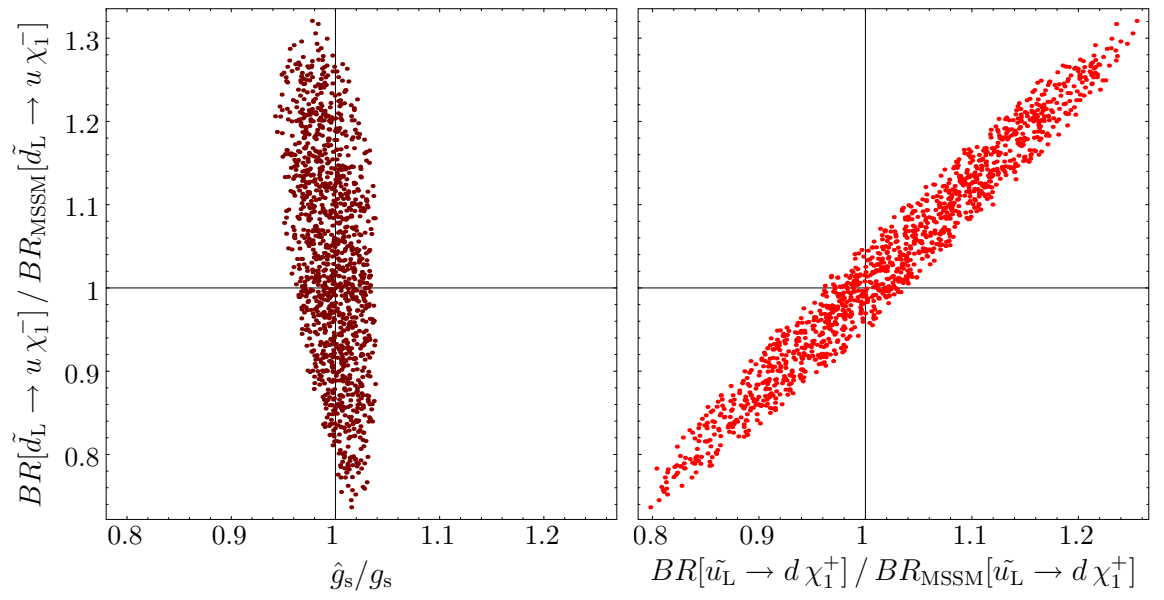

Figure 5: Determination of the SUSY-QCD Yukawa coupling $\hat{g}_{\mathrm{s}}$ and the L-squark branching ratios into charginos from a fit to the $p_{\mathrm{T} 3}$ distributions for positive $\left(\ell^{+} \ell^{+}\right)$and negative $\left(\ell^{-} \ell^{-}\right)$lepton pairs in the final state. The plots show the $1 \sigma$ statistical error regions corresponding to the fit method C.

\section{Conclusions}

A crucial consequence of supersymmetry is the identity of gauge couplings with the corresponding Yukawa couplings. Specifically in supersymmetric QCD, the quark-quark-gluon coupling and the quark-squark-gluino coupling are predicted to be equal [up to radiative corrections for SUSY scales different from the electroweak scale, described by renormalisation group running]. In the present analysis we have investigated how this relation can be tested through measurements at the Large Hadron Collider (LHC).

After identifying a clean signal of same-sign leptons, two or more jets and missing energy, that can be easily separated from Standard Model backgrounds, we have elaborated how this signal receives contributions from various squark and gluino production processes. Assuming a sufficiently large mass difference between gluino and squarks, it was further shown how gluino and squark production can be distinguished by analysing the transverse momentum distribution of additional jets. Since the gluino and squark production cross sections depend in different ways on the SUSY-QCD Yukawa coupling, this coupling can be extracted by measurements of the cross sections and jet distributions.

We have established analysis strategies that allow the extraction of the SUSY-QCD Yukawa coupling with varying degrees of model assumptions. In the most restricted case, it has been assumed that the complete decay pattern of the squarks in the MSSM is known, possibly from a high-energy $e^{+} e^{-}$collider. In this case one can perform a measurement of the total cross sections for squark-pair, squark-gluino and gluino-pair production at the LHC.

In a second step, we have extended the analysis to the situation when the squark branching ratios are not known, allowing for a more general structure of the theory, with isosinglet extensions of the MSSM, for instance. For relatively small mixing in the neutralino 
sector, several useful relations between different squark partial widths can be established based on gauge invariance, leaving only one branching ratio, $\tilde{u}_{L} \rightarrow \chi^{+}$to be determined internally within this LHC measurement. In case of large mixing in the neutralino sector, one ends up in general with three free parameters that nevertheless can be constrained from LHC data such that the impact on the measurement of the SUSY-QCD Yukawa coupling remains under good control.

For a specific scenario we have demonstrated the application of these methods. We have generated Monte-Carlo samples of the squark and gluino processes as well as the relevant backgrounds. After applying cuts to reduce the background, we have extracted the SUSY-QCD Yukawa coupling from appropriate fits to the simulated data. Including the most relevant expected systematic errors, we have found that the SUSY-QCD Yukawa coupling can be determined at LHC with a precision of a few per-cent, i.e. $3.4 \%$ in the most restricted method, and $7.5 \%$ in the most general method.

While these results are very encouraging, it should be pointed out that the experimental analysis and the expected quantitative precision will depend on the concrete supersymmetric scenario, i.e. the sparticle mass spectrum. The essential requirement is a light supersymmetric particle spectrum, that allows for sufficiently large event samples to perform advanced experimental analyses. The scenario studied here is representative for a large class in which gluinos are heavier than squarks, and gluino decays are distinguished by hard observable jets. Mutatis mutandis, the analyses can also be worked out for the reverse mass ratio, at the expense of controlling more involved cascade decays, though, which is beyond the scope of the present study. Nevertheless, our results demonstrate that precision measurements of the underlying structure of supersymmetric theories are feasible at the LHC if given requirements are met. It would be interesting to refine these studies in the future by including the impact of detector effects.

\section{Acknowledgments}

A.F. is supported in part by the Schweizer Nationalfonds. P.S. is supported by Fermi Research Alliance, LLC, under Contract No. DE-AC02-07CH11359 with the United States Department of Energy and M.S. in part by the Swiss Bundesamt für Bildung und Wissenschaft.

\section{References}

[1] J. Wess and B. Zumino, Supergauge transformations in four-dimensions, Nucl. Phys. B 70 (1974) 39.

[2] A. Freitas, A. von Manteuffel and P.M. Zerwas, Slepton production at $e^{+} e^{-}$and $e^{-} e^{-}$linear colliders, Eur. Phys. J. C 34 (2004) 487 [Addendum ibid. 40 (2005) 435] hep-ph/0310182] [Addendum hep-ph/0408341].

[3] S.Y. Choi, J. Kalinowski, G.A. Moortgat-Pick and P.M. Zerwas, Analysis of the neutralino system in supersymmetric theories, Eur. Phys. J. C 22 (2001) 563 hep-ph/0108117;

S.Y. Choi et al., Reconstructing the chargino system at $e^{+} e^{-}$linear colliders, Eur. Phys. J. C 14 (2000) 535 hep-ph/0002033. 
[4] H.-C. Cheng, J.L. Feng and N. Polonsky, Signatures of multi-TeV scale particles in supersymmetric theories, Phys. Rev. D 57 (1998) 152 hep-ph/9706476;

M.M. Nojiri, K. Fujii and T. Tsukamoto, Confronting the minimal supersymmetric standard model with the study of scalar leptons at future linear $e^{+} e^{-}$colliders, Phys. Rev. D 54 (1996) 6756 hep-ph/9606370.

[5] A. Freitas and P.Z. Skands, Determining the SUSY-QCD Yukawa coupling, JHEP 09 (2006) 043 hep-ph/0606121.

[6] W. Beenakker, R. Hopker, M. Spira and P.M. Zerwas, Squark and gluino production at hadron colliders, Nucl. Phys. B 492 (1997) 51 hep-ph/9610490];

T. Plehn, Prospino 2.0, online at pheno.physics.wisc.edu/ plehn/prospino/prosping.

[7] M.M. Nojiri and M. Takeuchi, The study of $s q_{L} s q_{L}$ production at LHC in the $l^{ \pm} l^{ \pm}$channel and sensitivity to other models, hep-ph/0701190.

[8] ECFA/DESY LC Physics Working Group collaboration, J.A. Aguilar-Saavedra et al., TESLA technical design report part III: physics at an $e^{+} e^{-}$linear collider, hep-ph/0106315.

[9] A. Alves, O. Eboli and T. Plehn, It's a gluino, Phys. Rev. D 74 (2006) 095010 hep-ph/0605067.

[10] B.C. Allanach et al., The snowmass points and slopes: benchmarks for SUSY searches, Eur. Phys. J. C 25 (2002) 113 hep-ph/0202233.

[11] B.C. Allanach, Softsusy: a C++ program for calculating supersymmetric spectra, Comput. Phys. Commun. 143 (2002) 305 hep-ph/0104145.

[12] T. Sjostrand, S. Mrenna and P. Skands, PYTHIA 6.4 physics and manual, JHEP 05 (2006) 026 hep-ph/0603175 online at http://projects.hepforge.org/pythia6/.

[13] T. Sjostrand and M. van Zijl, A multiple interaction model for the event structure in hadron collisions, Phys. Rev. D 36 (1987) 2019;

D. Field, CDF Note 6403 hep-ph/0201192; further recent talks available from webpage http://www.phys.ufl.edu/ rfield/cdf.

[14] F. Maltoni and T. Stelzer, Madevent: automatic event generation with MadGraph, JHEP 02 (2003) 027 hep-ph/0208156.

[15] P. Nason, S. Dawson and R.K. Ellis, The total cross-section for the production of heavy quarks in hadronic collisions, Nucl. Phys. B 303 (1988) 607; The one particle inclusive differential cross-section for heavy quark production in hadronic collisions, Nucl. Phys. $\mathbf{B}$ 327 (1989) 49 [Erratum ibid. 335 (1990) 260];

W. Beenakker, H. Kuijf, W.L. van Neerven and J. Smith, QCD corrections to heavy quark production in p p collisions, Phys. Rev. D 40 (1989) 54;

W. Beenakker, W.L. van Neerven, R. Meng, G.A. Schuler and J. Smith, QCD corrections to heavy quark production in hadron hadron collisions, Nucl. Phys. B 351 (1991) 507.

[16] ALTAS Technical Design Report, Vol 1, CERN/LHCC-99-15, chapt. 10;

E. Richter-Was, D. Froidevaux and L. Poggioli, ATLFAST 2.0 a fast simulation package for ATLAS, ATL-PHYS-98-131 (1998).

[17] J. Pumplin et al., New generation of parton distributions with uncertainties from global QCD analysis, JHEP 07 (2002) 012 hep-ph/0201195. 
[18] R. Lafaye, T. Plehn and D. Zerwas, Sfitter: SUSY parameter analysis at LHC and LC, hep-ph/0404282;

P. Bechtle, K. Desch, W. Porod and P. Wienemann, Determination of MSSM parameters from LHC and ILC observables in a global fit, Eur. Phys. J. C 46 (2006) 533 hep-ph/0511006.

[19] LHC/LC Study Group collaboration, G. Weiglein et al., Physics interplay of the LHC and the ILC, Phys. Rept. 426 (2006) 47 hep-ph/0410364.

[20] S.Y. Choi, D.J. Miller and P.M. Zerwas, The neutralino sector of the next-to-minimal supersymmetric standard model, Nucl. Phys. B 711 (2005) 83 hep-ph/0407209;

S.Y. Choi, H.E. Haber, J. Kalinowski and P.M. Zerwas, The neutralino sector in the $\mathrm{U}(1)$-extended supersymmetric standard model, hep-ph/0612218;

V. Barger, P. Langacker and G. Shaughnessy, Singlet extensions of the MSSM, AIP Conf. Proc. 903 (2007) 32 hep-ph/0611112].

[21] K. Desch, J. Kalinowski, G.A. Moortgat-Pick, M.M. Nojiri and G. Polesello, SUSY parameter determination in combined analyses at LHC/LC, JHEP 02 (2004) 035 hep-ph/0312069. 